\title{
Neutrino effective potential in a fermion and scalar background
}

\author{
José F. Nieves* \\ Laboratory of Theoretical Physics, Department of Physics, University of Puerto Rico, \\ Río Piedras, Puerto Rico 00936 \\ Sarira Sahu ${ }^{\dagger}$ \\ Instituto de Ciencias Nucleares, Universidad Nacional Autónoma de Mexico, Circuito Exterior, \\ C. U. A. Postal 70-543, 04510 Mexico DF, Mexico
}

(Received 14 August 2018; published 5 September 2018)

\begin{abstract}
We consider the neutrino self-energy in a background composed of a scalar particle and a fermion using a simple model for the coupling of the form $\lambda \bar{f}_{R} \nu_{L} \phi$. The results are useful in the context of dark matterneutrino interaction models in which the scalar and/or fermion constitute the dark matter. The corresponding formulas for models in which the scalar particle couples to two neutrinos via a coupling of the form $\lambda^{(\nu \nu \phi)} \bar{\nu}_{R}^{c} \nu_{L} \phi$ are then obtained as a special case. In the presence of these interactions there can be new contributions to the neutrino effective potential and index of refraction in the context of neutrino collective oscillations in a supernova and in the early Universe hot plasma before neutrino decoupling. The formulas obtained here can be used to estimate those effects and/or put limits on the model parameters based on the contribution that such interactions can have in those contexts. A particular feature of the results is that the contribution to the neutrino self-energy or effective potential in a neutrino background due to the $\nu \nu \phi$ coupling is proportional to the antineutrino-neutrino asymmetry $\left(n_{\bar{\nu}}-n_{\nu}\right)$, in contrast to the standard $Z$ contribution which is proportional to $\left(n_{\nu}-n_{\bar{\nu}}\right)$. Therefore the two contributions tend to cancel. If the cancellation is significant, it is conceivable that the $O\left(1 / m_{\phi}^{4}\right)$ terms give the dominant contribution. Alternatively, a limit can be set by requiring that the contribution of the $\nu \nu \phi$ interaction to the neutrino effective potential does not cancel the standard contribution in an appreciable way.
\end{abstract}

DOI: 10.1103/PhysRevD.98.063003

\section{INTRODUCTION}

The possible existence of complex scalars that interact with neutrinos via a coupling of the form

$$
L_{\text {int }}=\frac{1}{2} \lambda^{(\nu \nu \phi)} \bar{\nu}_{R}^{c} \nu_{L} \phi+\text { H.c. }
$$

has been explored recently [1-9]. Such scalars may be produced in terrestrial neutrino experiments, and constraints on their properties and interactions have been obtained from particle physics, astrophysics, and cosmology considerations in the works cited.

Here we note that such couplings would produce additional contributions beyond the standard ones to the neutrino index of refraction and effective potential when the neutrino propagates in a neutrino background. This

\footnotetext{
nieves@1tp.uprrp.edu

†sarira@nucleares.unam.mx
}

Published by the American Physical Society under the terms of the Creative Commons Attribution 4.0 International license. Further distribution of this work must maintain attribution to the author(s) and the published article's title, journal citation, and DOI. Funded by SCOAP ${ }^{3}$. occurs in the environment of a supernova, where it is now well known that the effect leads to the collective neutrino oscillations and related phenomena [10], and it can occur also in the hot plasma of the early Universe before the neutrinos decouple [11]. Therefore, further constraints on the $\nu \nu \phi$ couplings and/or the $\phi$ properties can be obtained by considering their effects in those contexts.

In a different line of development, models in which neutrinos interact with a scalar and a fermion via a coupling of the form

$$
L_{\text {int }}=\lambda \bar{f}_{R} \nu_{L} \phi+\text { H.c. }
$$

have been considered recently in the context of dark matterneutrino interactions [12,13]. Again, such interactions produce additional contributions to the neutrino effective potential when the neutrino propagates in a background of $\phi$ and $f$ particles.

In this paper we determine the effective potential of a neutrino or antineutrino that propagates in such backgrounds. To be specific, we calculate the neutrino selfenergy in a background of scalars $\phi$ and fermions $f$ due to the interaction given in Eq. (2). From the self-energy, the neutrino and antineutrino effective potential is then obtained. 
The corresponding formulas for the case of the neutrino and scalar background, with the couplings given in Eq. (1), are obtained as the special case in which $f_{R} \rightarrow \nu_{R}^{c}$. Our motivation is to provide a uniform treatment and present the results in a sufficiently general way such that they can be applied to the situations described and also adapted to others not considered here. For example, models in which sterile neutrinos have secret gauge interactions of the form $\bar{\nu}_{s} \gamma^{\mu} \nu_{s} A_{\mu}^{\prime}$ have been considered by several authors [14]. The formulas we obtain for the neutrino self-energy and effective potential, including the case of an anisotropic background that we consider, can be applied in the context of such models when a sterile neutrino propagates in a background of sterile neutrinos and $A^{\prime}$ bosons, with minor modifications.

In principle, the scalar-neutrino couplings can be of the form $\lambda_{i j}^{(\nu \nu \phi)} \bar{\nu}_{R i}^{c} \nu_{L j}$, involving various neutrino species. In writing Eq. (1) we are taking into account only the diagonal neutrino coupling and assuming the presence of only one scalar field. In the general case with more neutrino species in the background and nondiagonal neutrino- $\phi$ couplings, the density matrix formalism $[10,11]$ will come into play, which is outside the scope of the present work. It is worth mentioning that, despite the simplification we are making by considering only one neutrino type, the results reveal some interesting and potentially important features that can serve as a guide for considering the more general and realistic cases. A particular noteworthy feature is that the contribution to the neutrino self-energy or effective potential in the neutrino background due to this coupling is proportional to antineutrino-neutrino asymmetry $\left(n_{\bar{\nu}}-n_{\nu}\right)$, in contrast to the standard $Z$ contribution which is proportional to $\left(n_{\nu}-n_{\bar{\nu}}\right)$. Therefore, the two contributions tend to cancel. If the cancellation is significant, it is conceivable that the $O\left(1 / m_{\phi}^{4}\right)$ terms give the dominant contribution. As an example application, we use these results to indicate the constraint on $\lambda^{(\nu \nu \phi)} / m_{\phi}$ that can be placed based on the contribution that such interactions can have in the context of neutrino collective oscillations in a supernova.

In Sec. II, we review in a generic way the conventions that we use to determine the neutrino effective potential in a medium from the calculation of the self-energy. The calculation of the self-energy of the neutrino propagating in the background of fermions and scalars is undertaken in Sec. III. We consider various cases separately, depending on whether the background is isotropic or not, and various limits of the momentum distribution functions of the background particles. In Sec. IV, we use these results to calculate the effective potential under different physical conditions for a $\nu \phi$ background. We briefly summarize our results in Sec. V.

\section{DISPERSION RELATION AND EFFECTIVE POTENTIAL}

We consider the situation in which a neutrino propagates and interacts with the $f$ and $\phi$ background particles as

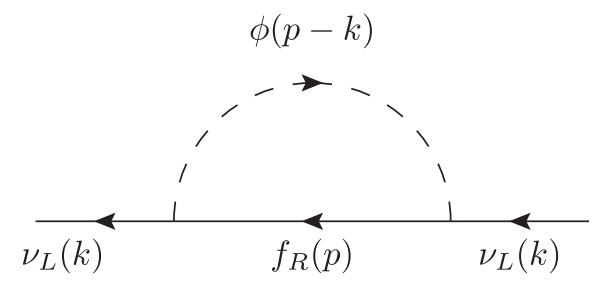

FIG. 1. Neutrino self-energy diagram in a background of fermions $f$ and scalars $\phi$.

illustrated in Fig. 1. For the purpose of determining the background contribution to the neutrino self-energy and dispersion relation, we treat the neutrino as a massless chiral particle. Denoting the neutrino momentum by $k^{\mu}=(\omega, \vec{\kappa})$, and the background contribution to the neutrino self-energy by $\Sigma^{(T)}$, the dispersion relations are obtained by solving

$$
\left(\not K-\Sigma^{(T)}\right) \psi_{L}=0
$$

The chirality of the neutrino interactions imply that $\Sigma^{(T)}$ is of the form

$$
\Sigma^{(T)}=V^{\mu}(\omega, \vec{\kappa}) \gamma_{\mu}
$$

The dispersion relations are then given by the solutions of

$$
\omega-V^{0}= \pm|\vec{\kappa}-\vec{V}|,
$$

which in general is an implicit equation for $\omega(\vec{\kappa})$. We consider two cases, according to whether the distribution functions are isotropic or not.

\section{A. Isotropic case}

In this case $V^{\mu}$ is of the form

$$
V_{\mu}=a(\omega, \kappa) k_{\mu}+b(\omega, \kappa) u_{\mu},
$$

where $u^{\mu}$ is the velocity four-vector of the background. In the frame in which the background is at rest, $u^{\mu}=(1, \overrightarrow{0})$, which we adopt from now on in this case. In terms of $a$ and $b$, Eq. (5) becomes

$$
(1-a) \omega-b= \pm(1-a) \kappa .
$$

As already mentioned, in the most general case this is an implicit equation for $\omega(\vec{\kappa})$. On the other hand, for small $a$ and $b$, the perturbative solutions around the vacuum solutions $\omega_{ \pm}(\vec{\kappa})= \pm \kappa$ are to the lowest order

$$
\omega_{ \pm}(\vec{\kappa})= \pm \kappa+b( \pm \kappa, \kappa)
$$

The neutrino and antineutrino dispersion relations, 


$$
\begin{gathered}
\omega_{\nu}(\vec{\kappa})=\omega_{+}(\vec{\kappa}), \\
\omega_{\bar{\nu}}(\vec{\kappa})=-\omega_{-}(-\vec{\kappa}),
\end{gathered}
$$

are then

$$
\omega^{(\nu, \vec{\nu})}(\vec{\kappa})=\kappa+V_{\mathrm{eff}}^{(\nu, \bar{\nu})}(\vec{\kappa}),
$$

where we have introduced the effective potentials $V_{\text {eff }}^{(\nu, \bar{\nu})}$, which in the present case are given explicitly by

$$
\begin{aligned}
& V_{\text {eff }}^{(\nu)}(\vec{\kappa})=b(\kappa, \kappa), \\
& V_{\text {eff }}^{(\bar{\nu})}(\vec{\kappa})=-b(-\kappa, \kappa) .
\end{aligned}
$$

\section{B. Anisotropic case}

In this case the $V^{\mu}$ cannot be decomposed in the form given in Eq. (6). We use Eq. (5) and assume that the equation can be solved perturbatively around the vacuum solutions $\omega_{ \pm}(\vec{\kappa})= \pm \kappa$. The solutions obtained in this way are then

$$
\begin{aligned}
& \omega_{+}(\vec{\kappa})=\kappa+V^{0}(\kappa, \vec{\kappa})-\hat{\kappa} \cdot \vec{V}(\kappa, \vec{\kappa}), \\
& \omega_{-}(\vec{\kappa})=-\kappa+V^{0}(-\kappa, \vec{\kappa})+\hat{\kappa} \cdot \vec{V}(-\kappa, \vec{\kappa}) .
\end{aligned}
$$

Identifying the neutrino and antineutrino dispersion relations by Eq. (9), they are given as in Eq. (10), where the effective potential in this case are

$$
\begin{aligned}
V_{\text {eff }}^{(\nu)}(\vec{\kappa}) & =V^{0}(\kappa, \vec{\kappa})-\hat{\kappa} \cdot \vec{V}(\kappa, \vec{\kappa}), \\
V_{\text {eff }}^{(\vec{\nu})}(\vec{\kappa}) & =-V^{0}(-\kappa,-\vec{\kappa})+\hat{\kappa} \cdot \vec{V}(-\kappa,-\vec{\kappa}) .
\end{aligned}
$$

Of course in the isotropic case, in which $V^{\mu}$ can be decomposed as in Eqs. (6), (13) reduces to Eq. (11).

\section{SELF-ENERGY IN THE $\boldsymbol{f} \phi$ BACKGROUND}

In this section we consider a neutrino propagating in a background of the fermion $f$ and complex scalar $\phi$ due to the interaction term in Eq. (2). The starting point is the calculation of the self-energy via the diagram shown in Fig. 1, from which the dispersion relations are determined as indicated in Sec. II.

The contribution of the diagram in Fig. 1 to the neutrino self-energy is given by

$$
-i R \Sigma L=|\lambda|^{2} \int \frac{d^{4} p}{(2 \pi)^{4}} i \Delta_{F}^{(\phi)}(p-k) R i S_{F}^{(f)}(p) L,
$$

where

$$
\begin{aligned}
i S_{F}^{(f)}(p) & =i S_{F 0}^{(\bar{\nu})}(p)-\Gamma_{f}(p), \\
i \Delta_{F}^{(\phi)}(p) & =i \Delta_{F 0}^{(\phi)}(p)+\Gamma_{\phi}(p),
\end{aligned}
$$

with

$$
\begin{aligned}
& \Gamma_{f}(p)=\not p 2 \pi \delta\left(p^{2}-m_{f}^{2}\right) \eta_{f}(p), \\
& \Gamma_{\phi}(p)=2 \pi \delta\left(p^{2}-m_{\phi}^{2}\right) \eta_{\phi}(p) .
\end{aligned}
$$

Using the label $x$ to stand for either $f$ or $\phi$, the functions $\eta_{x}(p)$ are given by

$$
\eta_{x}(p)=\theta\left(p^{0}\right) f_{x}\left(p^{0}, \vec{p}\right)+\theta\left(-p^{0}\right) f_{\bar{x}}\left(-p^{0},-\vec{p}\right),
$$

where $f_{x, \bar{x}}\left(E_{p}, \vec{p}\right)$ are the momentum distribution functions of the background particles and antiparticles, which may not necessarily be isotropic. For an isotropic thermal background, the distribution functions are

$$
\begin{aligned}
& f_{f, \bar{f}}\left(E_{f}\right)=\frac{1}{e^{\beta E_{f} \mp \alpha_{f}}+1}, \\
& f_{\phi, \bar{\phi}}\left(E_{\phi}\right)=\frac{1}{e^{\beta E_{\phi} \mp \alpha_{\phi}}-1} .
\end{aligned}
$$

Discarding the pure vacuum contribution to $\Sigma$ and denoting by $\Sigma^{(f, \phi)}$ the background-dependent part, we write

$$
\Sigma^{(f, \phi)}=\Sigma^{(f)}+\Sigma^{(\phi)},
$$

where

$\Sigma^{(f)}=-|\lambda|^{2} \int \frac{d^{4} p}{(2 \pi)^{3}} \frac{\not p}{(p-k)^{2}-m_{\phi}^{2}} \delta\left(p^{2}-m_{f}^{2}\right) \eta_{F}\left(p, \alpha_{f}\right)$,

$$
\Sigma^{(\phi)}=|\lambda|^{2} \int \frac{d^{4} p}{(2 \pi)^{3}} \frac{(\not p+\not k)}{(p+k)^{2}-m_{f}^{2}} \delta\left(p^{2}-m_{\phi}^{2}\right) \eta_{B}\left(p, \alpha_{\phi}\right) .
$$

We will evaluate $\Sigma^{(f, \phi)}$ in the following limiting cases. In the first case, to which we refer as the high temperature limit, we assume that the $f$ and $\phi$ backgrounds are isotropic, and also extremely relativistic so that their respective masses $m_{f, \phi}$ and chemical potentials $\alpha_{f, \phi}$ can be taken to be zero in Eqs. (20) and (21). In the second case we also assume the isotropic thermal distribution functions, but with the temperature and chemical potentials being such that the integrand in Eqs. (20) and (21) can be approximated by expanding them in powers of

$$
\Delta \equiv m_{\phi}^{2}-m_{f}^{2} .
$$

We refer to this as the heavy background limit. This also includes the case in which either $f$ or $\phi$ is massless, provided that the other particle is heavy enough. Finally we will consider a variant of the heavy background case, in which the distribution functions are not isotropic. By taking $f_{R}=\nu_{R}^{c}$ we can apply the results of any case, depending on whether $\phi$ is a light or heavy particle in the sense specified 
above, to the model in which $\phi$ couples to $\bar{\nu}_{R}^{c} \nu_{L}$ and consider situations in which a neutrino propagates in a neutrino background. Following the discussion in Sec. II, each $\Sigma^{(x)}$ is of the form

$$
\Sigma^{(x)}=V^{(x) \mu} \gamma_{\mu},
$$

and

$$
V_{\mu}=V_{\mu}^{(f)}+V_{\mu}^{(\phi)} .
$$

When the background is isotropic each $V^{(x) \mu}$ can be decomposed as

$$
V_{\mu}^{(x)}=a^{(x)} k_{\mu}+b^{(x)} u_{\mu}
$$

\section{A. High temperature limit}

Here we assume that $T$ and $\kappa$ are sufficiently large such that $f$ and $\phi$ can be taken to be massless. As we have already stated above, we then approximate the integrals in Eqs. (20) and (21) by setting $m_{f, \phi}$ and chemical potentials $\alpha_{f, \phi}$ to zero. In this case

$$
\begin{aligned}
\Sigma^{(f)} & =|\lambda|^{2} \int \frac{d^{4} p}{(2 \pi)^{3}} \delta\left(p^{2}\right) \frac{\not p}{(p+k)^{2}} \frac{1}{e^{|\beta p \cdot u|}+1}, \\
\Sigma^{(\phi)} & =|\lambda|^{2} \int \frac{d^{4} p}{(2 \pi)^{3}} \delta\left(p^{2}\right) \frac{(\not p+\not k)}{(p+k)^{2}} \frac{1}{e^{|\beta p \cdot u|}-1} .
\end{aligned}
$$

We will restrict ourselves to the dominant contribution in powers of $T$. In this case the term with the factor of $\not k$ in the integrand of Eq. (27) can be discarded and we can write

$$
\Sigma^{(x)}=|\lambda|^{2} I_{\mu}^{(x)} \gamma^{\mu}
$$

where

$$
I_{\mu}^{(f, \phi)}=\int \frac{d^{4} p}{(2 \pi)^{3}} \delta\left(p^{2}\right) \frac{p_{\mu}}{(p+k)^{2}} \frac{1}{e^{|\beta p \cdot u|} \pm 1} .
$$

The integrals in Eq. (29) are the same ones evaluated by Weldon for chiral fermions in a non-Abelian gauge theory [15] and also used in a previous discussion of the $\nu \nu \phi$ interaction model in Ref. [16]. Borrowing from those calculations, the results for $\Sigma^{(f, \phi)}$ can be written in the form of Eq. (25) with

$$
\begin{aligned}
& a^{(\phi)}=2 a^{(f)}=-\frac{|\lambda|^{2} T^{2}}{24 \kappa^{2}}\left[1-\frac{\omega}{2 \kappa} I\right], \\
& b^{(\phi)}=2 b^{(f)}=\frac{|\lambda|^{2} T^{2}}{24 \kappa}\left[\frac{\omega}{\kappa}-\frac{1}{2}\left(\frac{\omega^{2}}{\kappa^{2}}-1\right) I\right],
\end{aligned}
$$

where

$$
I \equiv \ln \left|\frac{\omega+\kappa}{\omega-\kappa}\right| .
$$

The total background-dependent part of the self-energy is then

$$
\Sigma^{(f \phi)}=a^{(f \phi)} \not k+b^{(f \phi)} \not l,
$$

with

$$
\begin{aligned}
& a^{(f \phi)}=-\frac{M^{2}}{\kappa^{2}}\left[1-\frac{\omega}{2 \kappa} I\right], \\
& b^{(f \phi)}=\frac{M^{2}}{\kappa}\left[\frac{\omega}{\kappa}-\frac{1}{2}\left(\frac{\omega^{2}}{\kappa^{2}}-1\right) I\right],
\end{aligned}
$$

where

$$
M^{2}=\frac{|\lambda|^{2} T^{2}}{16} .
$$

The corresponding dispersion relations in this case are given by the solutions of Eq. (7), with the coefficients $a, b$ given by Eq. (33). They are similar to the dispersion relations obtained by Weldon [15] for a chiral fermion in a non-Abelian gauge theory, which in particular have $\omega(\kappa \rightarrow 0)= \pm M$, and other interesting features $[17,18]$.

\section{B. Heavy background}

In this case we assume that either $f$ of $\phi$ is a heavy particle, in the sense that its mass is sufficiently larger than $T, \omega$, and $\kappa$, such that the denominators in the integrand in Eqs. (20) and (21) can be expanded in the form

$$
\begin{aligned}
& \frac{1}{(p+k)^{2}-m_{f}^{2}}=\frac{1}{\Delta}-\frac{2 p \cdot k+k^{2}}{\Delta^{2}}, \\
& \frac{1}{(p-k)^{2}-m_{\phi}^{2}}=-\frac{1}{\Delta}+\frac{2 p \cdot k-k^{2}}{\Delta^{2}},
\end{aligned}
$$

where $\Delta$ is defined in Eq. (22). Thus, $\Sigma^{(f, \phi)}$ are given as in Eq. (23) with

$$
\begin{aligned}
V_{\mu}^{(f)}(\omega, \vec{\kappa})= & |\lambda|^{2}\left(\frac{1}{\Delta}+\frac{k^{2}}{\Delta^{2}}\right) L_{\mu}^{(f)}-\frac{2|\lambda|^{2}}{\Delta^{2}} L_{\mu \nu}^{(f)} k^{\nu}, \\
V_{\mu}^{(\phi)}(\omega, \vec{\kappa})= & |\lambda|^{2}\left(\frac{1}{\Delta}-\frac{k^{2}}{\Delta^{2}}\right) L_{\mu}^{(\phi)}-\frac{2|\lambda|^{2}}{\Delta^{2}} L_{\nu}^{(\phi)} k^{\nu} k_{\mu} \\
& +|\lambda|^{2}\left(\frac{1}{\Delta}-\frac{k^{2}}{\Delta^{2}}\right) L^{(\phi)} k_{\mu}-\frac{2|\lambda|^{2}}{\Delta^{2}} L_{\mu \nu}^{(\phi)} k^{\nu},
\end{aligned}
$$

where

$\left(L^{(x)}, L_{\mu}^{(x)}, L_{\mu \nu}^{(x)}\right)=\int \frac{d^{4} p}{(2 \pi)^{3}} \delta\left(p^{2}-m_{x}^{2}\right) \eta_{x}(p)\left\{1, p_{\mu}, p_{\mu} p_{\nu}\right\}$ 
In order to obtain the effective potential from Eq. (13), we use the fact that, for $\omega=\kappa$, we have

$$
k^{\mu}=\kappa n^{\mu},
$$

where

$$
n^{\mu}=(1, \hat{\kappa}), \quad n^{2}=0 .
$$

From Eq. (13) the effective potentials are then given by

$$
V_{\mathrm{eff}}^{(\nu, \bar{\nu})}(\vec{\kappa})= \pm \frac{|\lambda|^{2}}{\Delta}\left(L_{1}^{(f)}+L_{1}^{(\phi)}\right)-\frac{2|\lambda|^{2}}{\Delta^{2}}\left(L_{2}^{(f)}+L_{2}^{(\phi)}\right),
$$

where the upper (lower) sign corresponds to the neutrino (antineutrino), respectively. In Eq. (40) the coefficients $L_{n}^{(x)}$ are defined as

$$
\begin{aligned}
& L_{1}^{(x)} \equiv L_{\mu}^{(x)} n^{\mu}, \\
& L_{2}^{(x)} \equiv L_{\mu \nu}^{(x)} n^{\mu} n^{\nu},
\end{aligned}
$$

and using the definitions in Eq. (37), the following formula follows:

$$
L_{n}^{(x)}=\frac{1}{2} \int \frac{d^{3} p}{(2 \pi)^{3}} E_{x}^{n-1}\left(1-\hat{\kappa} \cdot \vec{v}_{\vec{p}}\right)^{n}\left(f_{x}+(-1)^{n} f_{\bar{x}}\right) .
$$

For isotropic momentum distributions functions we can set $\vec{p} \rightarrow 0$ and $p^{i} p^{j} \rightarrow \frac{1}{3} \vec{p}^{2}$ in the integrands of $L_{1}^{(x)}$ and $L_{2}^{(x)}$, respectively, and the formulas reduce in that case to

$$
\begin{aligned}
L_{1}^{(x)} & =J_{1}^{(x)}, \\
L_{2}^{(x)} & =\frac{4}{3} J_{2}^{(x)}-\frac{1}{3} m_{x}^{2} J_{0}^{(x)},
\end{aligned}
$$

where

$$
J_{n}^{(x)}=\frac{1}{2} \int \frac{d^{3} p}{(2 \pi)^{3}} E_{x}^{n-1}\left(f_{x}+(-1)^{n} f_{\bar{x}}\right) .
$$

Using Eq. (43), the formulas given in Eq. (40) for the neutrino and antineutrino effective potential then reduce to

$$
\begin{aligned}
V_{\mathrm{eff}}^{(\nu, \bar{\nu})}(\vec{\kappa})= & \pm \frac{|\lambda|^{2}}{\Delta}\left(J_{1}^{(f)}+J_{1}^{(\phi)}\right)+\frac{2|\lambda|^{2} \kappa}{3 \Delta^{2}}\left(m_{f}^{2} J_{0}^{(f)}+m_{\phi}^{2} J_{0}^{(\phi)}\right. \\
& \left.-4 J_{2}^{(f)}-4 J_{2}^{(\phi)}\right),
\end{aligned}
$$

in the isotropic case. Similarly to Eq. (40), in Eq. (45) the upper (lower) sign corresponds to the neutrino (antineutrino), respectively. Introducing the number densities

$$
n_{x, \bar{x}}=g_{x} \int \frac{d^{3} p}{(2 \pi)^{3}} f_{x, \bar{x}}
$$

we have

$$
J_{1}^{(x)}=\frac{1}{2 g_{x}}\left(n_{x}-n_{\bar{x}}\right),
$$

where

$$
g_{f}=2, \quad g_{\phi}=1 .
$$

In anticipation to the application of these formulas in the next section we must remember that for chiral neutrinos

$$
g_{\nu}=1 \text {. }
$$

It is straightforward to obtain explicit formulas for the integrals $J_{0,2}^{(x)}$ in a number of cases. For example,

$$
\begin{aligned}
J_{n}^{(x)}= & \frac{1}{2 g_{x}}\left(n_{x}+(-1)^{n} n_{\bar{x}}\right) \\
& \times \begin{cases}m_{x}^{n-1} & (\text { NR limit }) \\
\frac{1}{2}(n+1) ! \beta^{1-n} & \text { (Maxwell - BoltzmanER limit })\end{cases}
\end{aligned}
$$

in the nonrelativistic (NR) or the Maxwell-Boltzman extremely relativistic (ER) limits.

\section{IV. $\nu \phi$ BACKGROUND}

In this section we use the results obtained in Sec. III to discuss the effects of the interaction given in Eq. (1) on a neutrino propagating in a neutrino- $\phi$ background. It is useful to remember that in the relevant diagram for this case, as shown in Fig. 2, the internal fermion that corresponds to the $f_{R}$ fermion line in Fig. 1 is the antineutrino $\nu_{R}^{c}$. As a result of this, the formulas obtained in Sec. III can be adapted to this case provided we identify $f, \bar{f} \rightarrow \bar{\nu}, \nu$ in the labels of the various physical quantities that refer to the background particles, such as the chemical potentials and particle number densities. In addition, we have to remember that for chiral neutrinos we must use $g_{\nu}=1$ in the formula for $J_{1}^{(\bar{\nu})}$ given in Eq. (47), as stated in Eq. (49).

\section{A. High temperature limit}

Denoting the background-dependent part of the selfenergy in this case by $\Sigma^{(\bar{\nu} \phi)}$ and writing

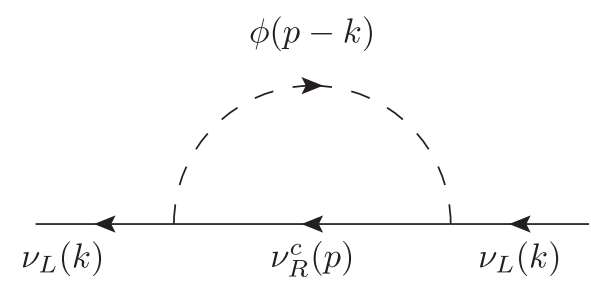

FIG. 2. Neutrino self-energy diagram in a background of neutrinos and scalars $\phi$. 


$$
\Sigma^{(\bar{\nu} \phi)}=a^{(\bar{\nu} \phi)} \not h+b^{(\bar{\nu} \phi)} \not h,
$$

the coefficients are given by the same expressions of Eq. (33), with

$$
M^{2} \equiv \frac{1}{16}\left|\lambda^{(\nu \nu \phi)}\right|^{2} T^{2}
$$

These are the results used also in a previous discussion of the $\nu \nu \phi$ interaction model in Ref. [16]. As already mentioned in Sec. III A, the dispersion relation for the neutrino in this case is similar to the dispersion relation obtained by Weldon [15] for a chiral fermion in a non-Abelian gauge theory. However, this case is not the one that is relevant to the situations where $m_{\phi}$, although assumed to be below the electroweak breaking scale, is assumed to be $\sim \mathrm{GeV}$, such as those considered in Ref. [1]. Thus we turn the attention to the case in which $\phi$ is sufficiently heavy such that its background density is negligible.

\section{B. Heavy $\phi$ limit}

For illustrative purposes we consider in some detail the isotropic case. From Eq. (11), neglecting the $\phi$ background terms and putting $m_{\nu}=0$, the $\nu \phi$ background contribution to the effective potentials is then

$$
\left(V_{\mathrm{eff}}^{(\nu, \bar{\nu})}(\vec{\kappa})\right)_{\nu \nu \phi}= \pm \frac{\left|\lambda^{(\nu \nu \phi)}\right|^{2}}{m_{\phi}^{2}} J_{1}^{(\bar{\nu})}-\frac{8\left|\lambda^{(\nu \nu \phi)}\right|^{2} \kappa}{3 m_{\phi}^{4}} J_{2}^{(\bar{\nu})} .
$$

The full background-dependent effective potential is then

$$
V_{\mathrm{eff}}^{(\nu, \bar{\nu})}(\vec{\kappa})=\left(V_{\mathrm{eff}}^{(\nu, \vec{\nu})}(\vec{\kappa})\right)_{\nu \nu \phi}+\left(V_{\mathrm{eff}}^{(\nu, \bar{\nu})}(\vec{\kappa})\right)_{Z}
$$

where $\left(V_{\mathrm{eff}}^{(\nu, \bar{\nu})}\right)_{Z}$ is the standard neutrino-background contribution from the $Z$ exchange and tadpole diagrams

$$
\left(V_{\mathrm{eff}}^{(\nu, \bar{\nu})}(\vec{\kappa})\right)_{Z}= \pm 4 \sqrt{2} G_{F} J_{1}^{(\nu)}
$$

Equation (55) follows, e.g., from the formulas obtained in Ref. [19]. As shown there, the contribution of those two diagrams to the neutrino self-energy can be written in the form of Eqs. (23) and (25), with

$$
\begin{aligned}
\left(b^{(Z)}\right)_{\text {tadpole }} & =4 \sqrt{2} G_{F} \sum_{f} X_{\nu} J_{1}^{(f)}, \\
\left(b^{(Z)}\right)_{Z-\text { exchange }} & =2 \sqrt{2} G_{F} J_{1}^{(\nu)},
\end{aligned}
$$

where the sum runs over all the fermion species in the background and $X_{f}$ is the (vector) neutral-current coupling of the fermion. In Eq. (55) we are including only the neutrino background contribution, and using $X_{\nu}=\frac{1}{2}$. Remembering Eqs. (47) and (49),

$$
J_{1}^{(\bar{\nu})}=-J_{1}^{(\nu)}=\frac{1}{2}\left(n_{\nu}-n_{\bar{\nu}}\right),
$$

we then have

$$
\begin{aligned}
V_{\text {eff }}^{(\nu, \bar{\nu})}(\vec{\kappa})= & \pm\left(2 \sqrt{2} G_{F}-\frac{\left|\lambda^{(\nu \nu \phi)}\right|^{2}}{2 m_{\phi}^{2}}\right)\left(n_{\nu}-n_{\bar{\nu}}\right) \\
& -\frac{8\left|\lambda^{(\nu \nu \phi)}\right|^{2} \kappa}{3 m_{\phi}^{4}} J_{2}^{(\bar{\nu})} .
\end{aligned}
$$

\section{Discussion}

In a core collapse supernova, neutrinos are trapped in the neutrino sphere and slowly diffuse out. These neutrinos have self-interactions that lead what is known as collective neutrino oscillations [10]. These phenomena can also occur in the hot plasma of the early Universe [11] before the neutrinos decouple. In the presence of the type of neutrinoscalar interaction we have considered, the collective oscillations could be modified.

A striking and surprising result is that the standard contribution $\left(V_{\text {eff }}^{(\nu, \bar{\nu})}\right)_{Z}$ on one hand, and $\left(V_{\text {eff }}^{(\nu, \bar{\nu})}\right)_{\nu \nu \phi}$ on the other, have opposite signs. Namely, they are proportional to $J_{1}^{(\nu)}=\frac{1}{2}\left(n_{\nu}-n_{\bar{\nu}}\right)$ and $J_{1}^{(\bar{\nu})}=\frac{1}{2}\left(n_{\bar{\nu}}-n_{\nu}\right)$, respectively. Thus, they tend to cancel. A limit can be set by requiring that the contribution of the $\nu \nu \phi$ interaction to the neutrino self-energy does not cancel the standard contribution in an appreciable way. In symbols we can express this as

$$
\left(V_{\mathrm{eff}}^{(\nu)}\right)_{\nu \nu \phi}<\left(V_{\mathrm{eff}}^{(\nu)}\right)_{Z}
$$

which requires

$$
m_{\phi}>\frac{\lambda^{(\nu \nu \phi)}}{g} m_{Z}
$$

Therefore, if $\lambda^{(\nu \nu \phi)} \sim O(1)$, this would imply $m_{\phi}$ must be larger than $\sim 3 m_{Z}$. In order to have $m_{\phi} \sim O(\mathrm{GeV})$, a small coupling $\lambda^{(\nu \nu \phi)} \sim 10^{-2}-10^{-3}$ would be required.

Another possibility is that the first term in Eq. (58), proportional to the neutrino-antineutrino asymmetry, is small, either because of the factor in parentheses and/or because the asymmetry is small. In such a case, the $O\left(1 / m_{\phi}^{4}\right)$ term in effective potential

$$
V_{\mathrm{eff}}^{(\nu, \bar{\nu})}(\vec{\kappa})=-\frac{8\left|\lambda^{(\nu \nu \phi)}\right|^{2} \kappa}{3 m_{\phi}^{4}} J_{2}^{(\bar{\nu})}
$$

would give the dominant contribution. For example, taking the Maxwell-Boltzman limit of the neutrino background from Eq. (50), this would give

$$
V_{\mathrm{eff}}^{(\nu, \bar{\nu})}(\vec{\kappa})=-\frac{2\left|\lambda^{(\nu \nu \phi)}\right|^{2} \kappa}{m_{\phi}^{4}} \frac{\left(n_{\nu}+n_{\bar{\nu}}\right)}{\beta},
$$


which is the same for the neutrino and antineutrino, and independent of the neutrino-antineutrino asymmetry in the background.

Although we have focused above on the case of isotropic thermal distributions of the neutrino background, similar considerations apply to the case of anisotropic distributions as well.

\section{CONCLUSIONS}

The existence of scalars that interact with neutrinos via couplings of the form $\lambda^{(\nu \nu \phi)} \bar{\nu}_{R}^{c} \nu_{L} \phi$ would produce additional contributions to the neutrino effective potential beyond the standard ones when the neutrino propagates in a neutrino background. This can occur in the environment of a supernova, and in the early Universe hot plasma before neutrino decoupling.

Motivated by studying the possible effects of such interactions in those contexts, we have considered a simple model in which a neutrino interacts with a scalar and a fermion, and we calculated the effective potential for a neutrino propagating in a background of those particles. The results are useful in the context of dark matter-neutrino interaction models in which the scalar and/or fermion constitute the dark matter, and are also applicable to the situations mentioned in which the fermion background is a neutrino background. We obtained the expressions for the neutrino effective potential that can be applied to different situations and background conditions.

As a specific application we considered the case of the neutrino background, with the scalar particle being sufficiently heavy so that their background density is negligible. A noteworthy result in this case is that the effective potential experienced by the propagating neutrino in this background has the opposite sign to the effective potential due to the standard contribution from the $Z$-boson. By assuming that the standard model contribution dominates over the $\nu \nu \phi$ contribution, e.g., in the neutrino sphere of a core collapse supernova, we have obtained the limit on the parameter $\lambda^{(\nu \nu \phi)} / m_{\phi}$.

Although for definiteness we have restricted ourselves to consider the diagonal scalar-neutrino coupling, the results already reveal some potentially important features, e.g., the sign difference mentioned above, that can serve as a guide for considering more realistic or complicated models involving, e.g., more than one scalar particle or off-diagonal neutrino couplings.
[1] J. M. Berryman, A. de Gouvea, K. J. Kelly, and Y. Zhang, Lepton-number-charged scalars and neutrino Beamstrahlung, Phys. Rev. D 97, 075030 (2018).

[2] Y. Farzan, M. Lindner, W. Rodejohann, and X. J. Xu, Probing neutrino coupling to a light scalar with coherent neutrino scattering, J. High Energy Phys. 05 (2018) 066.

[3] G. J. Stephenson, Jr. and J. T. Goldman, Observable consequences of a scalar boson coupled only to neutrinos, arXiv:hep-ph/9309308.

[4] C. Boehm, A. Olivares-Del Campo, S. Palomares-Ruiz, and S. Pascoli, Phenomenology of a neutrino-DM coupling: The scalar case, arXiv:1705.03692.

[5] L. Heurtier and Y. Zhang, Supernova constraints on massive (pseudo)scalar coupling to neutrinos, J. Cosmol. Astropart. Phys. 02 (2017) 042.

[6] R. F. Sawyer, Bulk viscosity of a gas of neutrinos and coupled scalar particles, in the era of recombination, Phys. Rev. D 74, 043527 (2006).

[7] P. S. Pasquini and O. L. G. Peres, Bounds on neutrino-scalar Yukawa coupling, Phys. Rev. D 93, 053007 (2016); Erratum, Phys. Rev. D 93, 079902(E) (2016).

[8] S. F. Ge, M. Lindner, and W. Rodejohann, Atmospheric trident production for probing new physics, Phys. Lett. B 772, 164 (2017).

[9] V. Brdar, J. Kopp, J. Liu, P. Prass, and X. P. Wang, Fuzzy dark matter and nonstandard neutrino interactions, Phys. Rev. D 97, 043001 (2018).
[10] See e.g., H. Duan, G. M. Fuller, and Y. Z. Qian, Collective neutrino oscillations, Annu. Rev. Nucl. Part. Sci. 60, 569 (2010), and references therein.

[11] See e.g., Y. Y. Y. Wong, Analytical treatment of neutrino asymmetry equilibration from flavor oscillations in the early universe, Phys. Rev. D 66, 025015 (2002), and references therein.

[12] R. Primulando and P. Uttayarat, Dark matter-neutrino interaction in light of collider and neutrino telescope data, J. High Energy Phys. 06 (2018) 026.

[13] T. Franarin, M. Fairbairn, and J. H. Davis, JUNO sensitivity to resonant absorption of galactic supernova neutrinos by dark matter, arXiv:1806.05015.

[14] X. Chu, B. Dasgupta, M. Dentler, J. Kopp, and N. Saviano, Sterile neutrinos with secret interactions: Cosmological discord?, arXiv:1806.10629 and references therein.

[15] H. A. Weldon, Effective fermion masses of order $\mathbf{g T}$ in high-temperature gauge theories with exact chiral invariance, Phys. Rev. D 26, 2789 (1982).

[16] J. F. Nieves, Neutrinos in a medium, Phys. Rev. D 40, 866 (1989).

[17] H. A. Weldon, Particles and holes, Physica A (Amsterdam) 158, 169 (1989).

[18] V. V. Klimov, Yad. Fiz. 33, 1734 (1981) [Spectrum of elementary fermi excitations in quark gluon plasma, Sov. J. Nucl. Phys. 33, 934 (1981)].

[19] J. C. D'Olivo, J.F. Nieves, and M. Torres, Finitetemperature corrections to the effective potential of neutrinos in a medium, Phys. Rev. D 46, 1172 (1992). 